\title{
SUPER TRIMUONS AS POSSIBLE EVIDENCE OF SIX-FERMION INTERACTION
}

R. MĂNIK, L. PALGI, H. OIGLANE. SUPERTRIMOOONID KUI KUUE FERMIONI INTERAKTSIOONI OLEMASOLU VOIMALIK TOEND

Р. МЯННИК, Л. ПАЛЬГИ, Х, ЫИГЛАНЕ. СУПЕРТРИМЮОНЫ ҚАК ВОЗМОЖНОЕ СВИДЕТЕЛЬСТВО СУЩЕСТВОВАНИЯ ШЕСТИФЕРМИОННОГО ВЗАИМОДЕНСТВИЯ

(Presented by P. Kard)

A number of neutrino scattering events with three outgoing muons $\left(\mu^{-} \mu^{-} \mu^{+}\right)$have been reported by several experimental groups: the CaltechFermilab (CF) collaboration [1], the Fermilab-Harvard-PennsylvaniaRutgers-Wisconsin (FHPRW) [ $\left.{ }^{2,3}\right]$, and the CERN-Dortmund-Heidelberg-Saclay (CDHS) collaboration [ $\left.{ }^{4}\right]$. In Fermilab $\left[{ }^{2,3}\right]$ two «super» events with extraordinary large muon energies were found: the average energies for the «fast» $\mu^{-}$, «slow» $\mu^{-}$and $\mu^{+}$are 127,53 and $85 \mathrm{GeV}$, while for «ordinary» events these figures are 55,8 and $20 \mathrm{GeV}$. Other characteristics of super events are not unusual. From the FHPRW $\left[{ }^{2,3}\right]$ data the observed total trimuon rate is $5 \times 10^{-4}$ per neutrino interaction above $100 \mathrm{GeV}$, and the observed rate of super events is more than one in 10 trimuon events. Although such super events have not been detected at CERN $\left[{ }^{4}\right]$ they deserve special attention.

The possible origins of trimuon events have been examined in many theoretical papers. A comprehensive study of four possible modes of trimuon production - the «hadronic», the «leptonic», the «mixed», and the trident mode - is given by R. M. Barnett, L. N. Chang, and N. Weiss [5]. In the hadronic mode of trimuon production the two additional muons are coming from the hadronic vertex, in the leptonic mode from the leptonic vertex, and in the mixed mode one additional muon from each vertex. In the trident mode of trimuon production a photon is radiated from off shell muon or quark and is converted into a muon pair. Each of these modes contains several sources of trimuon production, but the differences between the particular sources within the given mode are relatively small when compared with the differences between the modes [5]. It is remarkable that no mode of trimuon production discussed in [5] has any possibility of producing super events at the observed rate $\left[{ }^{2,3}\right]$. A thorough analysis by C. H. Albright, J. Smith, and J. A. M. Vermaseren $\left.{ }^{6}\right]$ of trimuon production mechanisms gave similar results. This suggests some qualitatively different origin of super events. Here we regard super trimuons as possible evidence for direct six-fermion interaction $\left[{ }^{7}\right]$. 
An interaction Lagrangian of direct six-fermion interaction for producing trimuons is a product of a neutral and a charged leptonic currents, and a charged hadronic current. We give here a rough estimate of the coupling constant $G_{6}$ of six-fermion interaction, using the following formula for the cross section, of neutrino six-fermion interaction $\left[{ }^{8}\right]$ :

$$
\sigma_{6}=2.8 \cdot 10^{-7} G_{6}^{2}(2 m E)^{4},
$$

where $\sigma_{6}$ is the cross section of super trimuon production and $E$ is the lab energy of the incident neutrino. From the FHPRW group $[2,3]$ data we have $\sigma_{6}=5 \cdot 10^{-5} \sigma^{\mu}$, where $\sigma^{\mu}$ is the total charged current cross section for neutrinos on nucleons, which in the energy range $20-205 \mathrm{GeV}$

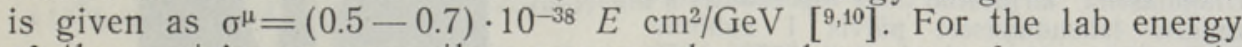
of the neutrino we use the average observed energy of super events $E \approx 250 \mathrm{GeV}$. From these data we get the constant of six-fermion interaction

$$
G_{6}=4 \cdot 10^{-9} \mathrm{GeV}^{-5} \text {. }
$$

Using the new data $\left[{ }^{9,10}\right]$ for the neutrino total cross section, a rough estimate $\left.{ }^{[}\right]$gives the upper limit

$$
G_{6}<7 \cdot 10^{-7} \mathrm{GeV}^{-5} .
$$

Because of the smallness of the constant of six-fermion interaction the cross section $\sigma_{6}$ violates unitarity at a very high energy. From Eqs. (1) and (2) we see that the unitarity violation begins at the energies of $4 \cdot 10^{4} \mathrm{GeV}$. Presumably the six-fermion interaction cross section exceeds the four-fermion interaction cross section not far before unitarity limit where the first-order calculation of $\sigma_{6}$ fails completely.

Assuming that super trimuons are created by six-fermion interaction, the creation of wrong-sign muons (single positive muons in neutrino interactions) is expected with the same rate as the creation of super trimuons. A search for single positive muon production for neutrino interactions has been made [11]. A cut at a visible energy of $100 \mathrm{GeV}$ gives an upper limit for the wrong-sign muon production relative to $\mu^{-}$ production of $1.6 \times 10^{-4}$. This does not exclude six-fermion interaction as a possible origin of super trimuons. The wrong-sign trimuons $\left(\mu^{+} \mu^{+} \mu^{-}\right)$ in the neutrino-induced six-fermion interaction are excluded and not observed.

\section{REFERENCES}

1. B a r is h, B. C., B a r t lett, J. F., et al., Phys. Rev. Lett., 38, № 11, 577-580 (1977).

2. Benvenuti, A., C 1 ine, D., et al., Phys. Rev. Lett., 38, № 20, 1110-1113 (1977).

3. B envenuti, A., B ob is ut, F., et al., Phys. Rev. Lett., 40, № 8, 488-491 (1978).

4. Holder, M., K n obloch, J., et al., Phys. Lett., B73, № 1, 105-108 (1978).

5. B a r nett, R. M., Ch a ng, L. N., We is s, N., Phys. Rev., D17, № 9, 2266-2275 (1978).

6. Albright, C. H., Smith, J., Vermaseren, J. A. M., In: FERMILABPUB-78/14-THY Jan. 1978, Batavia, Illinois, p. $1-28$.

7. Ericson, T., G 1 a s how, S. L., Phys. Rev., 133, 130-131 (1964).

8. Г ерштейн С. С., Фолом ешкин В. П., Ядер. физика, 15, вып. 3, 534-540 (1972).

9. B o s e t ti, P. S., D e de n, H., et al., Phys. Lett., B70, № 2, 273-277 (1977).

10. B a rish, B. C., B a rt lett, J. F., et al., Phys. Rev. Lett., 39, № 25, 1595-1598 (1977).

11. Holder, M., Knobloch, J., et al., Phys. Lett., B74, № 3, 277-281 (1978). 